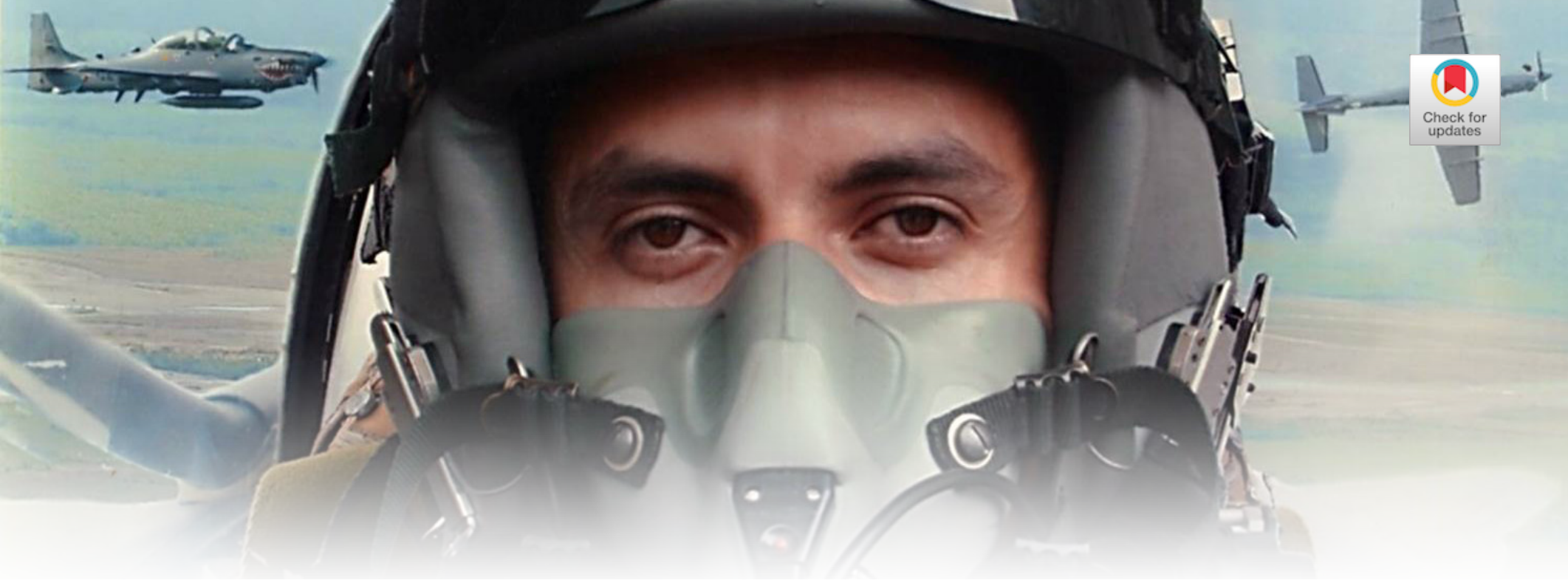

\title{
Aerocinetosis: más que enfermedad desadaptación
}

\author{
Motion Sickness: Maladjustment More Than Sickness²
}

\author{
María Alejandra Correa ${ }^{3}$ Luz Amparo Caputo ${ }^{4}$
}

CIENCIA Y PODER AÉREO

ISSN 1909-7050 | E-ISSN 2389-2468 |Volumen 9 | Enero-Diciembre de 2014 | Colombia | Pp. 17-25 Recibido: 20/02/2014 Aprobado evaluador interno: 08/09/2014 Aprobado evaluador externo: 17/10/2014

'Artículo científico original, derivado del proyecto: Aerocinetosis en las tripulaciones de la Fuerza Aérea Colombiana, autofinanciado por las investigadoras, con el apoyo del Centro de Medicina Aeroespacial.

${ }^{2}$ Original scientific article, from the project: Motion sickness on crews of the Colombian Air Force, self-funded by researchers, with support of the Center of Aerospace Medicine.

${ }^{3}$ Otorrinolaringóloga, Centro de Medicina Aeroespacial. Correo electrónico: correa10aleja@yahoo.com

Otorhinolaryngologist, Center of Aerospace Medicine. E-mail: correa10aleja@yahoo.com

${ }^{4}$ Fonoaudióloga. Especialista en Docencia Universitaria. Centro de Medicina Aeroespacial. Audiología Fuerza Aérea Colombiana. Correo electrónico: luzacaputo@hotmail.com Phonoaudiologist. Specialist in University Teaching. Center of Aerospace Medicine. Audiologist at Colombian Air Force. E-mail: luzacaputo@hotmail.com
Resumen: La cinetosis es una condición caracterizada por un grupo de síntomas como nauseas, vómito y sudoración fría, que ocurre cuando una persona es expuesta a un estímulo real o aparente de movimiento, que no es familiar. Este es un término genérico, se puede aplicar a los síntomas generados con el movimiento en el mar, aire, carro, natación, simulador de vuelo y el espacio. El comienzo del estímulo generado por el movimiento crea señales como las visuales, de la piel, de las articulaciones, los músculos, los receptores del sistema vestibular y gravito receptores; formando un conflicto entre la información, provocando la alteración. El tratamiento se orienta en la identificación de los síntomas, prevención de factores desencadenantes o predisponentes, y adaptabilidad al movimiento. La cinetosis es en sí un conflicto de sensaciones donde el cuerpo humano no está capacitado para definir e identificar la información percibida a menos que se entrene para lograr la desensibilización y habituación al movimiento. La aerocinetosis más que una patología es una desadaptación del organismo al movimiento durante el vuelo que genera un conflicto de sensaciones que el organismo no puede interpretar, pero puede ser entrenado y lograr el control y así continuar con su actividad laboral.

Palabras clave: Cinetocis, rehabilitación vestibular, vuelo.

Abstract: Motion sickness is a condition characterized by a group of symptoms such as nausea, vomiting, and cold sweating that occurs when a person is exposed to an unfamiliar, real or apparent, motion stimulus. It is a generic term, can be applied to the symptoms generated by movement in sea, air, car, swimming, flight stimulator, and the space. The beginning of the stimulus generated by the movement creates signals such as visual, of skin, joints, muscles, vestibular system's receptors and gravity receptors; originating a conflict between information, causing the alteration. The treatment is based in symptoms identification, prevention of triggering and underlying factors, and movement adaptability. Motion sickness is in fact a conflict of feelings where the human body is not capable of defining and identifying the information sensed, unless it is trained to achieve a decrease in sensitivity and habituation to movement. Motion sickness more than pathology is maladjustment of the organism to the movement during flight that generates feelings which cannot be interpreted but the person can be trained to achieve control, and in such a way continue with a normal life.

Key Words: Flight, Motion Sickness, Vestibular Rehabilitation. 


\section{Introducción}

La enfermedad del movimiento o cinetosis, hace referencia a una respuesta fisiológica universal normal, a una percepción anormal de movimiento (Shupak, 2006), se puede presentar en el movimiento durante viajes en barco o carro, en la piscina, en el espacio que es generado por las microgravedades. También, se encuentran en los simuladores de vuelo y en el vuelo, el cual se denomina Aerocinetosis (Ramírez, 2001).

El desarrollo de los síntomas en la enfermedad del movimiento es secuencial y varía según la intensidad del estímulo y la susceptibilidad del individuo. Los síntomas iniciales son molestias abdominales seguidas de náusea y malestar, posteriormente aparece palidez o enrojecimiento y sudoración fría, llevando a salivación, mareo, sensación de cuerpo caliente y vómito.

Horas después del estímulo pueden persistir síntomas como letargia, fatiga y adormecimiento. Cuando la persona continúa con los estímulos y presenta repetición de síntomas puede llegar a la apatía, depresión, disminución de la actividad cognitiva, aislamiento, alteración sicomotora originando el síndrome de sopite o avalancha (Shupak, 2006, y Golding, 2005) el cual puede estar aislado o asociado a la enfermedad del movimiento.

Cabe anotar que en el espacio se relaciona el evento agudo de cinetosis con la microgravedad que se presenta en el ambiente, iniciando síntomas de náuseas y gastrointestinales cuando ingresan a la órbita, estos síntomas se resuelven generalmente en un periodo entre las 30 y 48 horas después, pero pueden llegar a tener un rango hasta de 72 horas.

La aerocinetosis es una alteración frecuente en el personal de tripulantes en Colombia, no hay estudios ni parámetros para el manejo en la aviación militar y civil. En el Centro de Medicina Aeroespacial surgió la necesidad primero de analizar cómo y por qué se presentaba la aerocinetocis, segundo crear un programa de entrenamiento para reacondicionar a los tripulantes con el ánimo de continuar con su actividad laboral de vuelo.

\section{Método}

El artículo es producto del trabajo de investigación: Aerocinetosis desarrollado en el Centro de Medicina Aeroespacial desde el 2007, de enfoque mixto, de tipo descriptivo y comparativo; en el cual se efectuó una revisión bibliográfica sobre la entidad, tanto en las bases de datos internacionales, como el manejo que se hace en las Fuerzas Militares de otros países. Después se comparó con los síntomas de las tripulaciones de la Fuerza Aérea Colombiana lo que permitió generar una guía de manejo dirigida y adaptada a la población de tripulantes militares de Colombia.

\section{Significado operacional}

La enfermedad del movimiento o cinetosis es una condición que altera la habilidad del piloto en vuelo generando síntomas que lo distraen en la actividad laboral, perdiendo finalmente el control de la nave. Se presenta con más frecuencia en personal de entrenamiento de vuelo, a diferencia del equipo de vuelo y simuladores de vuelo. Inicialmente es manejado por el instructor, quien le da pautas al alumno, y generalmente son tomadas de experiencias vividas por ellos, proporcionando "remedios caseros".

La persona que presenta el evento le produce ansiedad, pérdida de confianza en sí mismo y en su actividad de vuelo, lo que aumenta la intensidad de los síntomas en algunos casos.

Los síntomas pueden ser típicos como: dolor de estómago, nauseas, mareo, somnolencia, vómito; y los síntomas no típicos como: visión borrosa, cefalea, dificultad de la concentración, dolor ocular. Se pueden presentar síntomas post-estímulo, como desórdenes posturales, sensación ilusoria del movimiento, destellos visuales, desorientación y mareo permanente; pueden durar desde 12 horas a una semana o más; lo cual impide la realización de sus actividades laborales.

Los pasajeros (tripulantes) también pueden presentar, cinetosis generándoles limitaciones en sus actividades laborales.

\section{Etiología}

El origen o teoría más aceptada de la enfermedad del movimiento, está dada por un conflicto en el procesamiento de la información, es un problema en el procesamiento de la información dentro de un sistema sensorial multimodal cuya función está determinada por el movimiento relativo individual y de su ambiente. Se ha llamado la hipótesis del Mismatch neural (Benson, 2000). El conflicto se puede originar dentro de un único sistema sensorial (Ejemplo: interacción de los canales semicirculares y los otolitos) o entre dos o más sistemas (Ejemplo: sistema visual y vestibu- 


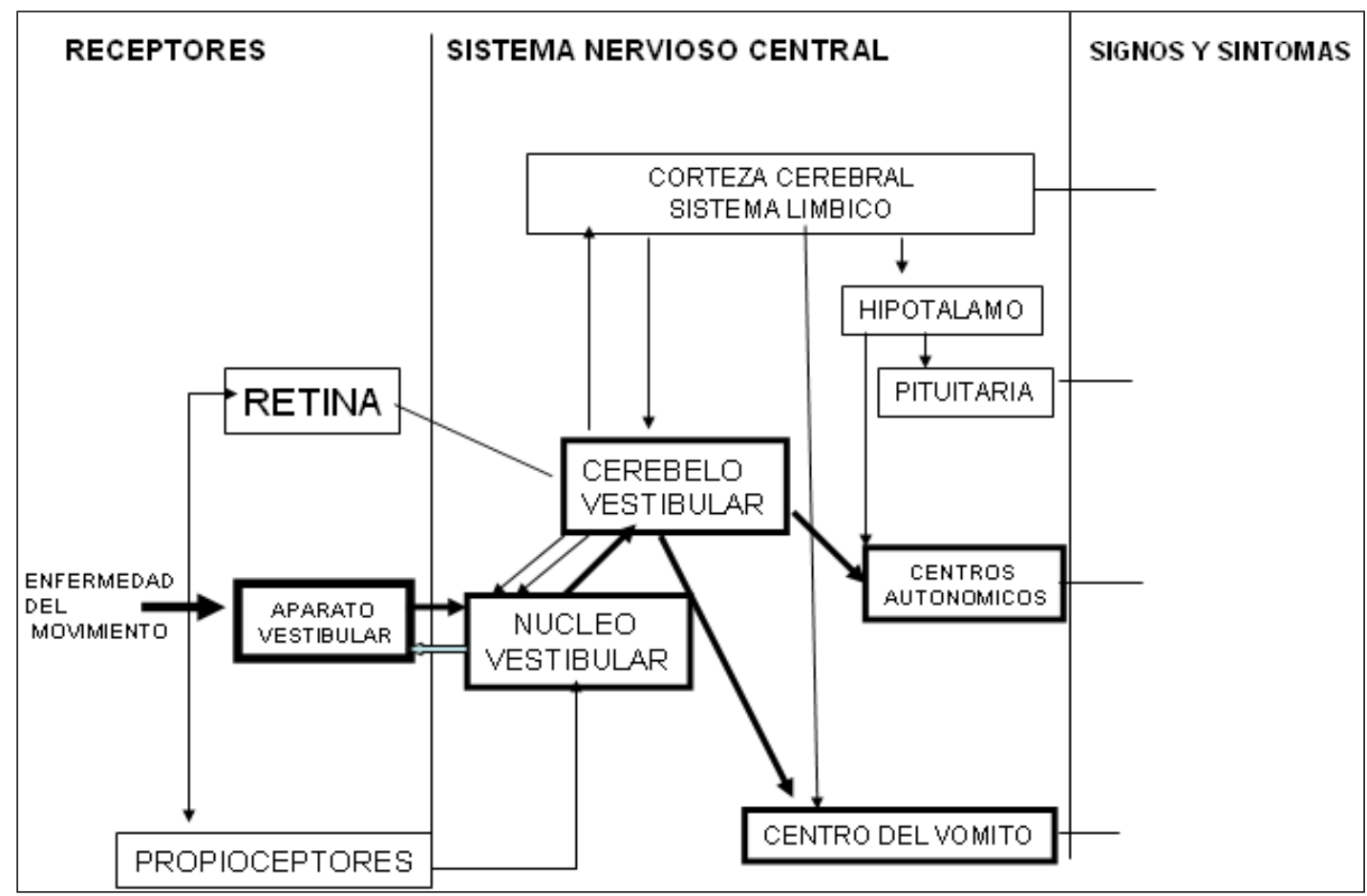

Figura 1. Estructuras Neurales comprometidas en la enfermedad del movimiento.

Fuente: Benson, Alan J. (2000). Capítulo 33 Motion sickness. Operacional Aviation Medicine, p. 458.

lar). El conflicto es seguido por unas respuestas anticipadas de los impulsos sensoriales y la habituación al movimiento generado (Benson, 2000) (Ver Figura 1).

Hay otras teorías que se pueden tener en cuenta para comprender la patogénesis de la enfermedad del movimiento. Wood y Graybiel (1977), lo explican por medio del modelo farmacológico, así las sinapsis centrales colinérgicas utilizan acetilcolina trasmitiendo la información del movimiento desde el sistema vestibular y terminando en los órganos de la ejecución. La transmisión neuronal es antagonizada por la sinapsis simpática las cuales usan epinefrina y norepinefrina (Shupak, 2006).

Treisman (1977), propone que los síntomas premonitorios generados por la enfermedad del movimiento son un biproducto accidental del sistema de protección del organismo, que identifica neurotoxinas, generando emesis y así se deshace de las toxinas (Shupak, 2006).

Von Baum Garten (1979) y otros, han sugerido una asimetría en la función de los otolitos entre los dos laberintos, lo explican con el cambio de la masa de las otoconias, siendo la causa de la enfermedad del movimiento. Las otoconias buscan contrarrestar el estímulo con el sistema ocular, en el momento del movimiento derecha- izquierda, donde son más sensibles cuando se encuentran con una exposición pasiva o con variaciones de la fuerza gravitacional. Shupak (2006), Benson (2000) Riccio y Stoffregen (1991), basan su hipótesis en la inestabilidad del control postural, esta teoría aumenta la incidencia por la frecuencia del movimiento externo, lo que interfiere con la onda natural, aumentando la actividad espontánea del movimiento.

Ebenholtz (1994), sugiere que la enfermedad del movimiento es secundaria a los movimientos oculares que están regulados por el núcleo vestibular. El movimiento induce nistagmos resultados de la tracción de los músculos extra oculares con la activación del reflejo ocular, cardíaco y estimulación del nervio vago. También, esta teoría se extiende a una posible estimulación del nervio trigémino en el segmento anterior del ojo y explicaría la asociación de la enfermedad del movimiento y migraña.

Bless (2000) y otros, lo soportan en la teoría subjetiva del conflicto vertical, caracterizado por la condición en que el movimiento vertical es tomado por la información dada por los ojos y el sistema vestibular y el propioceptivo pueden tener una variación del movimiento vertical. Shupak, (2006), Finley (1996) y otros, han postulado las diferencias de las respuestas autonómicas individuales al estrés inherente en el campo psicológico, fisiológico y ambiental. Se ha estudiado el receptor 
adrenérgico (alfa2- AR) del cromosoma 10 asociándolo con la respuesta periférica y central del sistema autónomo asociándolo con la respuesta periférica y central del sistema autónomo, incrementando los síntomas y signos en la enfermedad del movimiento. (Shupak, 2006).

\section{Correlación fisiológica}

Se asocia con la actividad fisiológica especialmente con el sistema nervioso autónomo, que causa la sudoración y palidez causada por la vasoconstricción de los vasos cutáneos y la vasodilatación de los vasos profundos, alteración de la motilidad y el tono intestinal. Se ha detectado incremento de la secreción hormonal de la pituitaria con aumento de la hormona antidiurética, hormona adrenocorticotropica, epinefrina y norepinefrina, con lo cual lleva a aumento de la frecuencia cardiaca y tensión arterial. A nivel de microgravedades dado en los vuelos espaciales se encuentra alteración en los niveles de la hormona antidiurética.

\section{Incidencia}

La enfermedad del movimiento es una respuesta de adaptación a un ambiente de movimiento no conocido, hay diferencias entre los individuos y su susceptibilidad.

La incidencia reportada en el trasporte civil es del $1 \%$, la mayoría se presenta en la penetración en huracanes o en turbulencias (Benson, 2000). En la aviación militar se manifiesta durante el entrenamiento del personal de vuelo. Según la Fuerza Aérea Royal es del 39\%, el $15 \%$ presentan síntomas severos que deben abandonar el vuelo. US Navy Flight tiene una incidencia del 13.5\%, severos en un $5.9 \%$. Se ha descrito que de un $55 \%$ a un $83 \%$ de los estudiantes de vuelo han tenido algún tipo de síntomas en el primer vuelo. En los estudios en entrenamiento de vuelos espaciales se ha encontrado una incidencia de $67 \%$ y de estos el 13\% tienen síntomas severos (Mudin, 2011).

Se ha encontrado factores que podrían aumentar la aparición de síntomas pero no son concluyentes como orientación del cuerpo, gravedad, dirección del estímulo, la baja frecuencia de la oscilación lineal, el tipo de movimiento y otros.

La considerable variabilidad de los síntomas entre los individuos que presentan el mal del movimiento, se sugiere explicarlo a través de unos conceptos que son:
- $\quad$ Receptividad: se refiere a la susceptibilidad individual del sistema nervioso y psicológico.

- Adaptabilidad: rango en el cual la persona se adapta a un movimiento atípico.

- Retención o memoria de adaptación.

Ejemplo: si un individuo que tiene gran capacidad de adaptación, retentiva y receptividad se habituará fácilmente al movimiento (Ver Figura 2).

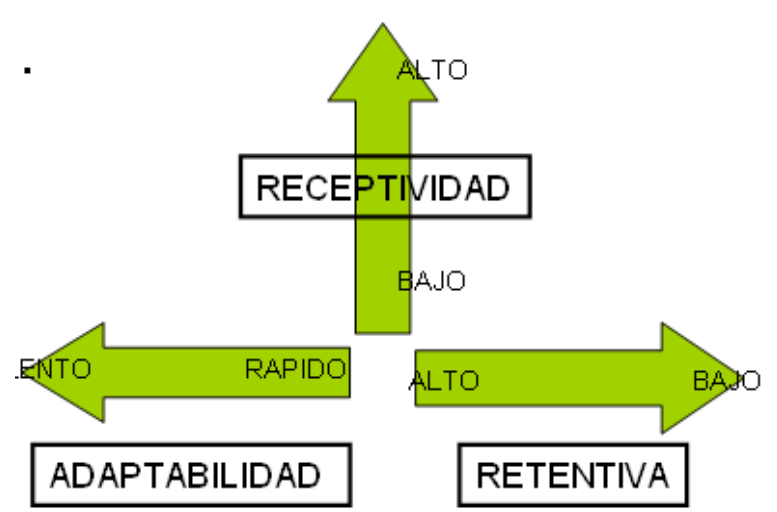

Figura 2. Factores que determina la susceptibilidad de la enfermedad del movimiento.

Fuente: Benson, Alan J. (2000). Capítulo 33 Motion sick-

ness. Operacional Aviation Medicine, p. 467.

\section{Clasificación}

La enfermedad del movimiento se puede clasificar según la intensidad de los síntomas en grados así:

- Grado I Síntomas mínimos: sensación de náusea, dispepsia y mareo leve, no interfiere con la actividad que este realizando.

- Grado Il: síntomas moderados, náuseas, mareo, sudoración, sialorrea. Puede seguir realizando la actividad, pero tiene que dejar de dar movimiento fuertes, vueltas.

- Grado III: síntomas severos, aumenta la intensidad de los síntomas del grado ll y no puede seguir con la actividad que realiza en el momento.

- Grado IV: síntomas incapacitantes, emesis activa, postración, cefalea, compromiso del estado de conciencia, desorientación; detiene cualquier actividad, se busca asistencia médica.

\section{Tratamiento}

Es un manejo interdisciplinario se orienta en varias entidades: 


\section{Adaptación}

Es la más potente medida terapéutica. Se debe tener un estímulo permanente regular y repetitivo para desencadenar la adquisición y mantenimiento de adaptación protectora ante el estímulo del movimiento. Hay diferencias entre la susceptibilidad y el rango de adaptación. Teniendo en cuenta la exposición y la intensidad del estímulo, lo que genera memoria del evento que origina las medidas adaptativas (Bless, 2000).

Hay técnicas investigadas, para el personal pre vuelo en los procesos de adaptación, especialmente para los viajes espaciales, en donde a través de la coriolisis, aceleraciones lineales, angulares e ingravidez, generan habituación del personal al ambiente. Se encuentran equipos de entrenamiento vestibular como el DISO, GAT II y Gyroflight que se usa para la estabilización de los gravito receptores y contribuye a la orientación espacial. Estas estimulaciones también se realizan post vuelos para habituar a las personas que presentan síntomas durante el vuelo. Se determinan en ellas todas las respuestas que se presenten (Previc, 2004).

Se presenta con gran frecuencia el mal del movimiento al iniciar el entrenamiento de vuelo en tripulantes. Este mejora, con los procesos de adaptación a estímulos repetitivos, se ha encontrado un 5\% que falla en este proceso de adaptación protectora. En la "Royal Air Force" Benson (2000) realiza hace más de 20 años, un entrenamiento en simuladores que exponen al tripulante a movimientos repetitivos durante 1 a 3 semanas con 2 exposiciones al día, con aumento progresivo en la intensidad del estímulo. En la Fuerza Aérea de los Estados Unidos la desensibilización utiliza un feedback con técnicas de relajación con estimulación de coriolisis pasiva, asociado a soporte de psicoterapia para manejar la ansiedad generada por el evento. Con una efectividad de un $85 \%$. Otros factores que influyen en la generación del conflicto son los relacionados con la aeronave.

\section{Factores de la aeronave}

La enfermedad del movimiento, se incrementa o disminuye, según la frecuencia de oscilación de la aeronave, esto es importante en el diseño de la aeronave y su dinámica de vuelo. Una aeronave que responde a una ráfaga de perturbación de bajo frecuencia $(<0.5$ $\mathrm{Hz}$ ) presentará movimientos menos deseables. Ejemplo: un huracán lleva a alta frecuencia y el "roll danés" induce la generación del autocontrol del piloto. También, es importante la estabilidad postural del piloto con buen soporte de la cabeza y del cuello a la silla. Un papel im- portante representa el control de los movimientos de la cabeza y el cuerpo durante el vuelo. Otros son los factores ambientales como: calor, olores indeseables y presión que exacerban la aparición de la enfermedad del movimiento.

Partiendo del significado de habituación, como un proceso central de aprendizaje, en el que se disminuye la susceptibilidad a las condiciones de movimiento durante varias semanas y varias veces al día. Se recomienda un entrenamiento en que se aplique este concepto con movimientos elípticos, optoquinéticos y otros, asociados a la simulación de la aeronave con su microambiente, para que el personal identifique los síntomas individuales que le genera y los pueda manejar.

\section{Medicación}

Durante años se han buscado medicamentos para la prevención de la enfermedad del movimiento. Ninguno tiene la posibilidad de prevenirla, y todos tienen efectos secundarios como sedación, depresión del sistema nervioso central, boca seca y/o visión borrosa. Por lo cual es contraindicado que un piloto tome algún medicamento de estos durante sus periodos de vuelo.

El vómito es una de las manifestaciones más incapacitarte, ya que produce la pérdida de su actividad durante el vuelo, además puede llevar a deshidratación, trastorno hidroelectrolítico, que requiere manejo de líquidos para la hidratación y uso de medicamentos como metroproclamida u ondancetron para el control del vomito.

\section{Programa de entrenamiento en el Centro de Medicina Aeroespacial}

En el Centro de Medicina Aeroespacial, se trabaja sobre una guía de tratamiento generada, posterior a una revisión bibliográfica y de las Fuerzas Aéreas de otros países, sobre el manejo de aerocinetosis. Se han tomado los casos de aerocinetosis que se consultaron en el CEMAE de la Fuerza Aérea desde el 2007, los cuales fueron y son manejados según la guía que se estructuró. La cual cuenta con el diagnóstico, tratamiento y prevención. El manejo se dividió en dos fases:

\section{Fase I}

Se denominó fase de caracterización de la población susceptible a la enfermedad del movimiento. En esta se capacita a las personas acerca de esta alteración y se inicia la prevención a través del personal médico de las unidades aéreas, con psicólogos e instructor de 
CIENCIA Y PODER AÉREO | Revista Científica de la Escuela de Postgrados de la Fuerza Aérea Colombiana | Vol. 9 | Enero - Diciembre de 2014

vuelo. Inicialmente se observó que las indicaciones iniciales son dadas por el instructor y el médico de la unidad aérea.

Basados en la bibliografía se buscó instaurar en las unidades de entrenamiento, programas de exposición continúa al movimiento por lo menos una hora al día, varias semanas seguidas (Gans, 1999), antes de iniciar la actividad de vuelo.

Acompañado de apoyo psicológico, se citan algunas recomendaciones alimentarias y de actividad física así:

- Tomar alimentos 2 o 3 horas antes del vuelo.

- Evitar alimentos ácidos.

- Evitar bebidas carbonatadas.

- Evitar la cafeína.

- Evitar comidas grasosas.

- Evitar cigarrillo.

- Mantener una buena hidratación.

- Tener descanso adecuado.

- Ejercicio periódico continuo.

- Usar ropa adecuada.

Cuando el paciente no mejore de los síntomas o persisten en las actividades de vuelo se remiten al CE$M A E$, donde se inicia la Fase 2.

\section{Fase II}

Se realiza la evaluación del paciente por parte de un grupo interdisciplinario: médico de aviación, otorrinolaringología, audiología y psicología. Se realiza la evaluación médica por otorrinolaringología, exámenes audiológicos, se toman paraclínicos, entre ellos están: cuadro hemático, perfil lipídico, glicemia y TSH. Al tiempo se inicia el tratamiento, el cual consta de 4 puntos principales, estas indicaciones son dirigidas por otorrinolaringología y audiología.

1. Técnica de respiración y relajación: se explica al tripulante la forma correcta de realizar la respiración costo-diafragmática la cual debe utilizar en su vida diaria y le ayudara a prevenir la cinetosis. Se dan técnicas de relajación de segmentos del cuerpo especialmente extremidades inferiores y superiores con tensión y distensión para lograr un mejor manejo.

2. Apoyo Psicológico: se cimienta en el apoyo psicológico de la ansiedad generada durante el vuelo.
3. Entrenamiento vestibular: se basa en la readaptación o habituación al movimiento, se utiliza la silla de Barany inventada por el húngaro Robert Barany, la cual tiene como objetivo habituar al tripulante con movimientos repetitivos asociado a desencadenar desorientación espacial, con el fin de lograr una adaptación a los diferentes sensaciones que se pueden presentar en vuelo como: somato giratoria y coriólisis. Se realiza de la siguiente forma: se ubica al tripulante en la silla, se ajusta con el cinturón, se le explica que va a girar, se le colocan unas gafas oscuras las cuales van a inhibir el estímulo visual. Se gira inicialmente lo más despacio posible hasta lograr la mayor velocidad dependiendo de la tolerancia del paciente; primero con la cabeza quieta y después se inician movimientos de cabeza en todas las direcciones arriba - abajo, derecha -izquierda y diagonales en forma progresiva, buscando la habituación al movimiento. Se detiene cuando el paciente no puede tolerarlo o hasta completar una duración máxima de 20 minutos en cada sesión. Dependiendo del grado de cinetosis se realizan diferentes sesiones entre dos días a una semana, mínimo dos diarias (Ver Tabla 1).

4. Educación al paciente: guía de recomendaciones al paciente (Ver Anexo 1).

Al finalizar los días de entrenamiento se evalúa nuevamente al tripulante por parte de otorrinolaringología, determinando su condición final y retorna a la unidad para continuar su actividad de vuelo, con un seguimiento de apoyo por parte del personal médico de la unidad.

Se le dan indicaciones, en caso de presentar síntomas durante el vuelo, podrá mitigar los síntomas realizando las siguientes actividades:

- Usar técnica de respiración costo diafragmático.

- Evitar movimientos de la cabeza bruscos, se recomienda primero girar los ojos seguida de la cabeza.

- Mantener control del timón de la aeronave.

- Sí los pies se colocan fríos, se recomienda movimientos de ellos para mantener la circulación.

Se le entrega recomendaciones al paciente. 
Tabla N. 1 Grados de aerocinetosis y entrenamiento

\begin{tabular}{llll}
\hline \multicolumn{1}{c}{ GRADO I } & \multicolumn{1}{c}{ GRADO II } & \multicolumn{1}{c}{ GRADO III } & \multicolumn{1}{c}{ GRADO IV } \\
\hline $\begin{array}{l}\text { Síntomas leves: náuseas, } \\
\text { dispepsia, mareo leve }\end{array}$ & $\begin{array}{l}\text { Síntomas moderados: } \\
\text { además de lo anterior, } \\
\text { sudoración y sialorrea. }\end{array}$ & $\begin{array}{l}\text { Síntomas severos: } \\
\text { aumenta la intensidad de } \\
\text { los síntomas y palidez. }\end{array}$ & $\begin{array}{l}\text { Síntomas incapacitantes: } \\
\text { emesis, desorientación, } \\
\text { alteración conciencia. }\end{array}$ \\
\hline Apoyo psicología & Apoyo psicología & Apoyo psicología & Apoyo psicología \\
\hline Ejercicios de respiración y & $\begin{array}{l}\text { Ejercicios de respiración y } \\
\text { relajación }\end{array}$ & $\begin{array}{l}\text { Ejercicios de respiración y y } \\
\text { relajación }\end{array}$ & Ejercicios de respiración y \\
\hline 2 sesiones en silla de & 5 sesiones en silla de & 7 sesiones en silla de & 10 sesiones en silla de \\
Barany & Barany & Barany & Barany \\
Un día & Dos días y medio & Tres días y medio & Cinco días \\
\hline
\end{tabular}

Fuente: Elaborado por las autoras.

\section{Conclusiones}

La aerocinetosis es una respuesta fisiológica a una percepción anormal del movimiento real o aparente durante el vuelo. No se tiene claridad acerca de su etiología, se conoce que es incapacitante, limitando la actividad laboral. En el medio aeronáutico encontramos que la mayor aparición es en el personal de entrenamiento, cambio de equipo y uso de simuladores.

En el Centro de Medicina Aeroespacial de la Fuerza Aérea Colombiana, se encontró una incidencia de 14 pacientes que consultaron entre 2007 al 2014, de estos el $80 \%$ presentaron aerocinetosis grado IV, según la clasificación descrita. Se observó un $80 \%$ al cambiar de equipo y el $20 \%$ restante al iniciar su entrenamiento de vuelo.

La edad promedio de aparición es entre 22 a 26 años, correspondiente a los grados de Subteniente y Teniente; lo cual está asociado a las etapas de formación, coincidiendo con las teorías y diferentes documentos que mencionan que este tipo de desadaptación se presenta al comienzo de la instrucción. Se efectúo el manejo a los tripulantes según el grado de 3 a 5 días con dos terapias diarias (7 am-11am), con la toma y evaluación de los laboratorios, las sugerencias por psicología, la evaluación y entrenamiento por otorrinolaringología y fonoaudiología, finalmente la educación al paciente explicándole la patología, dando las indicaciones y las recomendaciones a seguir. Los 14 tripulantes regresaron a sus actividades de vuelo sin recurrencia del cuadro hasta el momento.

Con el inicio de este protocolo de manejo se ha generado la información para capacitar a los médicos de las unidades, durante los cursos de Medicina Aeroespacial que imparte el Centro de Medicina Aeroespacial, logrando el correcto manejo de la Aerocinetosis en el personal de tripulantes, en la Fase I en las bases y la remisión al CEMAE cuando los síntomas continúan y es necesario el manejo de la Fase II.

Se optimizó el reintegro de los tripulantes a sus actividades resaltando la importancia de la salud operacional y la seguridad aérea.

El resultado final e importante es la generación de un conocimiento científico; crear estadísticas de la población colombiana para iniciar la caracterización de las patologías en nuestra población y capacitar al personal médico en el manejo de estos eventos y la educación del paciente sobre el tema a través de la guía de recomendaciones.

\section{Referencias}

Benson, Alan J. (2000). Capítulo 33 Motion sickness. Operacional Aviation Medicine, pp. 455-471. USA.

Bless, W. Bos, J. y Kruit, H. (2000). Neuro-ophtalmoloy and neurootology. Motion sickness, pp. 19 - 24.

Braithwaite, M. (2004). Capítulo 8. Spatial Desorientation in Aviation, pp. $323-347$.

Fonseca, W. (2000). Habituación sensorial con ejercicios de gimnasia en la profilaxis de la aerocinetosis de la Fuerza Aérea de Brasil, pp. 100-109.

Gans, R. (1999). Protocols and programs vestibular rehabilitation. USA.

Golding, J., y Gresty, M. (2005). Motion Sickness. Neuro-ophtalmoloy and neuro-otology, pp. 29 - 32 USA.

Mudin, L. (2011). Managing Motion Sickness. BMJ, pp. 1-7 USA.

Previc, F. y Ercoline, W. (2004). Spatial disorientation in Aviation. Instituto Americano de aeronáutica y astronomía. USA.

Reschke, Millard, F., y Harm, Deborah L. (2010). Chapter XXII Neurophysiologic aspects: Space Motion Sickness. Physiology adaptation to space flight. 3 Edition, pp. 228-255. USA. 
Shopak. A, Gordon, C. (2006). Motion Sickness: Advance in phatogenesis, predition, prevention and treatment. Aviation space and environmental medicine, vol 77, pp. 1213-1220. USA.

TC. Ramírez, M.. (2001). Manual: el malestar en vuelo de la Fuerza Aérea de los Estados Unidos, pp. 79- 87. Fuerza Aérea de los Estados Unidos de América.

\section{Anexo 1. \\ Guía de Recomendaciones Aerocinetosis}

\section{¿Qué es la aerocinetosis?}

$\mathrm{NO}$ es una enfermedad. Es un conflicto sensorial que se genera durante el movimiento de la aeronave, se presenta síntomas como: náuseas, sudoración, dolor abdominal, palidez, aumento de la respiración, taquicardia hasta vomito. Según los síntomas se clasifica en:

- Grado I: Síntomas leves, sensación de náusea, dispepsia y mareo leve, no interfiere con la actividad que esté realizando.

- Grado II: Síntomas moderados, nauseas, mareo, sudoración, sialorrea. Puede seguir realizando la actividad, pero tiene que dejar de dar movimiento fuertes, vueltas.

- Grado III: Síntomas severos, aumenta la intensidad de los síntomas del grado II y no puede seguir con la actividad que realiza en el momento.

- Grado IV: Síntomas incapacitantes, emesis activa, postración, cefalea, compromiso del estado de conciencia, desorientación; detiene cualquier actividad, se busca asistencia médica.

\section{Tener en cuenta:}

- Tomar alimentos 2 o 3 horas antes del vuelo.

- Evitar alimentos ácidos

- Evitar bebidas carbonatadas (gaseosas o con gas)

- Evitar la cafeína

- Evitar comidas grasosas.

- Evitar cigarrillo

- Mantener una buena hidratación

- Tener descanso adecuado

- Ejercicio periódico continúo

- Usar ropa adecuada

\section{¿Qué pueden hacer durante el vuelo para mitigar} los síntomas?

- Usar técnica de respiración costo diafragmático.
- Evitar movimientos de la cabeza bruscos, se recomienda primero girar los ojos seguida de la cabeza.

- Mantener control del timón de la aeronave.

- Si los pies se colocan fríos se recomienda movimientos de ellos para mantener la circulación.

\section{Ejercicios vestibulares}

Realizarlo 2 veces al día por 10 minutos, cerca de la cama acompañado si es posible, suspenderlos cuando realice todos los ejercicios y ninguno le dé sensación de mareo. (Ver Figura 3).

Nivel. 1: Ejercicios oculares; cabeza mantenida inmóvil

Puede hacerse en la cama si el paciente stá agudamente enfermo o sentado). Uirar arriba, luego abajo, primero tespacio y luego de prisa, manteniendo a cabeza inmóvil. 20 veces.

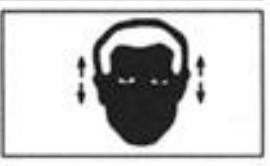

Mirar de un lado a otro, manteniendo la cabeza inmóvil, primero despacio y luego deprisa. 20 veces.

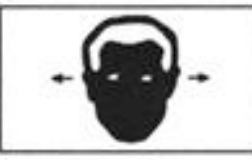

Focalizar un dedo de la mano, estando el brazo extirado, moverlo entonces desde unos treinta y ocho centímetros hacia la nariz y luego alejarlo otra vez. 20 veces.

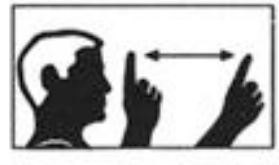

Nivel 2: Movimientos de la cabeza y los ojos

(Sentado o en cama).

Flexión y extensión de la cabeza con los ojos abiertos, despacio y luego deprisa. 20 veces.

Inclinación de lado a lado de la cabeza, despacio y después deprisa. 20 veces. Cuando mejore el desequilibrio, se repetirá el nivel 2 con los ojos cerrados.
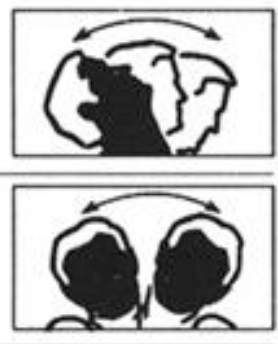

Nivel 3: Movimientos de brazo y cuerpo: sentado

TSi previamente se realizaron en la cama, repetir estando sentado los niveles 1 y 2).

Encogerse de hombros. 20 veces. Hacer círculos con los hombros. 20 veces.

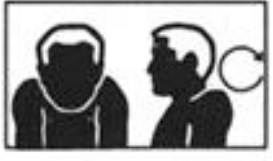

Girar la cintura a la derecha y luego a la izquierda. 20 veces.

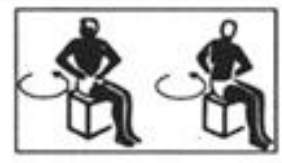


Inclinarse hacia adelante para coger un objeto de la cama o del suelo si se está sentado en una silla. Incorporarse y después agacharse para reponer el objeto. Repetir 20 veces.

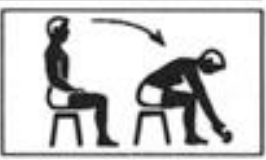

Girar la cabeza de lado a lado, dos giros lentos y luego rápido. Esperar unos segundos y hacer tres giros rápidos. Cuando prosiga la mejona, repetir con los ojos cerrados.

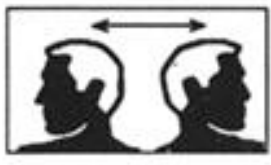

\section{Nivel. 4: Levantándose}

Repetir el nivel 3 levantado.

Movilizarse, de estar sentado, ponerse de pie con los ojos abiertos. 20 veces. Repetir con los ojos cerrados.

Tirar una pelota de tenis o algo similar de mano a mano, asegurándose que la bola pasa por encima del nivel del ojo. 20 veces.

Inclinarse hacia adelante y pasar la
bola de mano a mano detrás de una
rodilla. 20 veces. Repetir con los ojos
cerrados.
cerrados.

Cambiar de sentado a de pie, volverse una vez y entonces, sentarse otra vez. Repetir 10 veces.

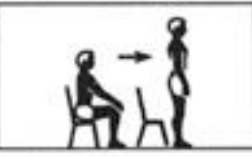

\section{Nivel. 5: Trasladándose}

Caminar a través de la habitación, alrededor de una silla y entonces, volver atrás a través de la habitación. Tras 10 repeticiones, intentarlo con los ojos cerrados.

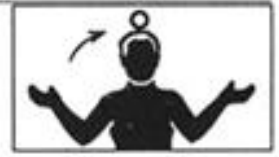

Estando en grupo o con un familiar o amigo, practicar tirar una bola grande atrás y hacia adelante y luego con el paciente caminando en círculo alrededor de la persona se le lanza la bola.
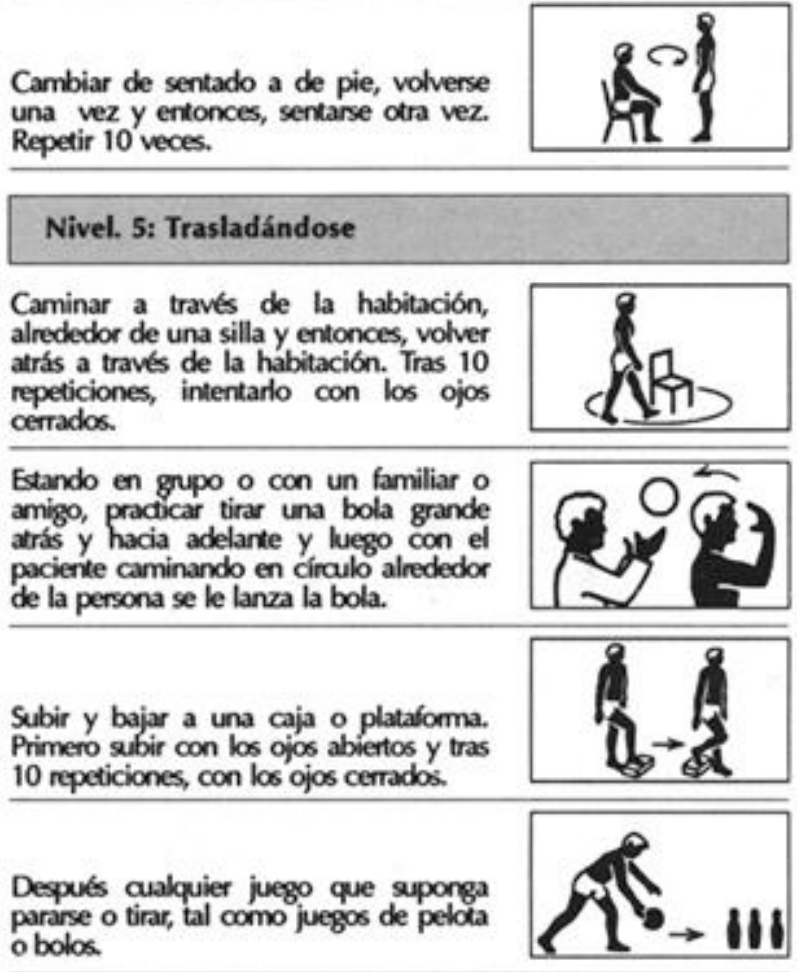

Si el paciente va a realizar los ejercicios en su casa con un amigo, deberá proporcionársele una cuartilla conteniendo instrucciones claras y una explicación de las razones de los ejercicios.

Figura 3. Ejercicios vestibulares.

Fuente: ICS. Disponible en: http://www.ics.gencat.cat/3clics/main.php desadaptación. Ciencia y Poder Aéreo, Vol. 9 (1). Pp. 17-25. 